\title{
Strates
}

STRATES Matériaux pour la recherche en sciences sociales

$12 \mid 2006$

Nouvelles tensions impériales et recompositions en Europe centrale, orientale, et CEI

\section{L'espace Baltique-mer Noire}

\section{Bruno DRWESKI}

\section{(2) OpenEdition \\ 1 Journals}

\section{Édition électronique}

URL : http://journals.openedition.org/strates/1902

DOI : $10.4000 /$ strates. 1902

ISSN : 1777-5442

Éditeur

Laboratoire Ladyss

\section{Édition imprimée}

Date de publication : 31 décembre 2006

ISSN : 0768-8067

Référence électronique

Bruno DRWESKI, «L'espace Baltique-mer Noire », Strates [En ligne], 12 | 2006, mis en ligne le 19 juillet 2007, consulté le 08 septembre 2020. URL : http://journals.openedition.org/strates/1902 ; DOI :

https://doi.org/10.4000/strates.1902

Ce document a été généré automatiquement le 8 septembre 2020.

Tous droits réservés 


\section{L'espace Baltique-mer Noire}

\section{Bruno DRWESKI}

1 Géographiquement, les pays situés sur l'isthme entre la mer Baltique et la mer Noire et reliant la masse continentale de l'Eurasie avec la péninsule européenne constituent un « entonnoir » qui a favorisé les contacts entre les différentes populations habitant cet espace transitoire. Pour la Russie, vu de l'Est, l'espace Baltique-mer Noire a constitué souvent la voie de passage de la plupart des envahisseurs (chevaliers teutoniques, Polonais, Napoléon, Guillaume II, Hitler). Pour l'Allemagne, vu de l'Ouest, cet espace représente un terrain de déstabilisation potentielle (hordes tatares, bolchevisme). Pour les habitants du lieu, les envahisseurs de l'Ouest et de l'Est se sont croisés sur leur propre territoire.

2 Historiquement, aucune langue unique ni aucune foi commune n'a jamais dominé seule cette région mais du XIV e siècle au XVIII ${ }^{e}$ siècle, soit pour la plus longue partie de son histoire, cet espace a fait partie d'un État commun, pluraliste et diversifié par nature, la République polono-lithuanienne. C'est ce croisement d'influences et cette histoire commune qui se sont prolongés au xIX $x^{\mathrm{e}}$ siècle et une partie du $\mathrm{Xx}^{\mathrm{e}}$ siècle, par le biais du maintien d'une structure féodale marquée par l'hégémonie de la noblesse polonisée et catholique sur des paysans d'origines et de confessions différenciées. Cette structure sociale était complétée par la concentration sur ce territoire de la quasi-totalité des Juifs européens entre la fin du Moyen Age et le début du XIX ${ }^{e}$ siècle.

3 Aujourd'hui, après le reflux de l'impérialisme allemand et la désagrégation de l'hégémonie russe, les nations de l'espace Baltique-mer Noire se sont toutes retrouvées au sein d'État-nations à base ethnique. Cette situation nouvelle va-t-elle se stabiliser ou ne constitue-t-elle qu'une transition vers la formation de nouvelles structures supranationales?

4 L'isthme situé entre la mer Baltique et la mer Noire a toujours été une zone d'échanges et d'affrontements. Les grandes puissances ont toujours voulu maitriser ce " couloir d'invasions ». C'est pourquoi l'État russe, dont les centres névralgiques sont situés dans le voisinage immédiat de l'espace Baltique-mer Noire, a eu comme objectif fondamental de verrouiller ce territoire. La confrontation entre les intérêts stratégiques de la Russie, poussée à la centralisation du fait de son étendue, et les désirs des populations locales 
accoutumées à résister à tout embrigadement explique une partie des drames qui les ont frappées depuis deux siècles.

Du XIII ${ }^{\mathrm{e}}$ siècle au XVIII ${ }^{\mathrm{e}}$ siècle, l'espace Baltique-mer Noire a échappé à la domination d'États centralisés et s'est trouvé englobé au sein de la communauté étatique polonolithuanienne qui au départ, a assuré à des populations d'origines ethniques et religieuses différentes (catholiques, orthodoxes, protestants, juifs, musulmans) le respect de leurs diversités. L'unité de cet ensemble reposait sur la création d'un espace économique lié à l'exportation du blé vers l'Europe occidentale et au refus de l'intolérance religieuse qui, à l'Ouest, mena à l'expulsion des juifs, aux guerres de religion et à l'Inquisition, pendant qu'à Moscou, l'Église orthodoxe devenait le fondement unique de l'État. Mais le régime de démocratie nobiliaire polono-lithuanien ne sut pas s'adapter à l'émergence de la bourgeoisie et de la paysannerie. La généralisation du "second servage " affaiblit l'État polono-lithuanien et permit à la Russie, à l'Autriche et à la Prusse de se partager l'espace Baltique-mer Noire. La politique centralisatrice des tsars causa des drames : suppression des libertés cosaques en Ukraine, insurrections polonaises du $\mathrm{XIX}^{\mathrm{e}}$ siècle, pogroms contre les juifs, etc. L'expansion du capital, puis de l'Etat allemand frappa avec brutalité les populations de l'espace Baltique-mer Noire, d'abord par la politique de pillage généralisé appliquée pendant la Première Guerre mondiale puis par l'exploitation sans limites, les déportations massives, la terreur et le génocide pendant la Seconde Guerre mondiale.

L'État soviétique fut presque dès l'origine amené à tenir compte des données géostratégiques qui avaient guidé la politique des tsars, ce qui explique son acharnement à contrôler l'espace Baltique-mer Noire. Les dirigeants soviétiques renoncèrent cependant, après leur échec devant Varsovie en août 1920, à annexer directement la Pologne, dont ils craignaient l'influence "contaminatrice» sur un régime socialiste que Lénine reconnaissait comme trop frustre. Sous Staline, l'URSS annexa en revanche l'ensemble des territoires biélorussiens et ukrainiens puis la façade baltique de la Russie, élargie à la Prusse orientale dans la foulée de la victoire sur l'agresseur allemand. La politique soviétique de développement économique accéléré alla de pair avec la répression impitoyable de toute tendance potentiellement centrifuge: liquidation de l'autonomie des républiques soviétiques après 1929, collectivisation et grande famine, déportations massives (Grecs et Tatars de Crimée, Allemands, Polonais, Baltes, etc.). Mais ces territoires connurent un développement économique et social rapide. Les républiques baltes jouirent d'un niveau de vie bien supérieur à la moyenne soviétique. La Biélorussie atteignit puis dépassa le niveau économique de la Russie et l'Ukraine concentrait en 1991 environ 40 \% de la puissance industrielle de toute l'URSS ${ }^{1}$. Ces sociétés crurent alors qu'il serait possible de déserrer l'étau du système soviétique centralisé tout en gardant les avantages acquis. C'était négliger le fait que ces économies s'étaient développées grâce au vaste ensemble soviétique qui permettait d'accéder à moindre prix aux matières premières, de bénéficier de réseaux d'innovations technologiques et de profiter de clients « captifs »à l'échelle de «l'empire».

7 Aujourd'hui encore, les anciennes républiques des confins occidentaux de l'URSS ne peuvent espérer une véritable reprise économique sans coopération avec l'espace postsoviétique. Après l'Histoire, c'est à la Géographie de reprendre ses droits. L'espace Baltique-mer Noire a besoin de redevenir un carrefour d'échanges économiques, diplomatiques, culturels et humains entre l'Europe maritime et la masse continentale 
de l'Eurasie. C'est pourquoi nous considérons qu'il existe un ensemble Baltique-mer Noire, indépendamment du fait que certains pays qui le composent reconnaissent formellement y appartenir par le biais de la Communauté des États Indépendants (CEI) (Biélorussie, Ukraine, Moldavie) et d'autres non (pays Baltes). On peut même rattacher à cet ensemble la Pologne pour des raisons à la fois historiques, géographiques et économiques.

8 Les pays baltes en particulier n'ont pas cessé avec la disparition de l'URSS d'être la façade maritime de la Russie et de la Biélorussie. L'Estonie et la Lettonie comptent en plus entre un tiers et une moitié de "russophones ", ce qui contribue à les "amarrer " au monde post-soviétique. La minorité russophone est moins nombreuse en Lituanie, mais l'enclave russe de Kaliningrad contribue au maintien de liens entre Moscou et Vilnius. Après avoir été coupées pendant plusieurs décennies de tout contact extérieur échappant au strict contrôle de Moscou, les sociétés de l'espace Baltique-mer Noire ont voulu mettre après 1991 "le cap à l'Ouest ». Mais les attentes envers l'Occident n'ont été que partiellement satisfaites, ce qui explique pourquoi on constate aujourd'hui un regain d'intérêt pour la reprise de contacts avec les anciens partenaires soviétiques. Cela vaut pour les russophones mais aussi pour une partie des couches populaires marginalisées comme pour beaucoup de « jeunes loups » à la recherche de débouchés à l'abri de la concurrence des grandes firmes occidentales. Les entrepreneurs russes ou occidentaux cherchent aussi à exploiter la situation de l'espace Baltique-mer Noire pour y établir des firmes jouant sur le transit.

9 Le maintien de l'Otan, alors que le pacte de Varsovie acceptait de se dissoudre pacifiquement, a cependant contribué à réveiller les divergences dans cette zone de l'Europe, aujourd'hui sommée de choisir entre «l'ancien Occident» structuré et le «nouvel Orient» en recomposition. Cela explique le malaise qui traverse les populations qui ont cru être libérées de la nécessité de faire des choix catégoriques perçus comme mutilants. Entre l'Occident remilitarisé sous l'égide des États-Unis depuis les guerres de Yougoslavie et d'Irak, et la Russie s'accrochant aux symboles de son ancienne puissance, les peuples de l'espace Baltique-mer Noire ont dû renoncer à la réalisation rapide du rêve associant développement économique, progrès social, frontières ouvertes et sécurité. Ce rêve avait donné sa force aux accords d'Helsinki mais le «nouvel ordre mondial » promis ne s'est pas réalisé après le démantèlement du « rideau de fer ». La généralisation de l'insécurité, la dégradation des conditions de vie et l'adoption progressive par la Pologne, sous la pression de l'Union européenne (UE), des normes de Shengen contribuent à créer de nouveaux murs et à délégitimer la «transition" vers un modèle de société devenu de plus en plus flou à mesure qu'on s'en rapproche.

La Biélorussie, voie de passage entre Moscou et l'Occident

10 Alors que, dans le passé, les grandes voies d'invasion passaient par les steppes ukrainiennes, le développement de Moscou a entraîné la création de voies nouvelles traversant le territoire biélorussien. Ce territoire occupe une position stratégique fondamentale, comme on l'a constaté sous Napoléon et pendant les deux guerres mondiales. Depuis 1991, la Biélorussie est redevenue un lieu de confrontation potentiel puisque les puissances de l'otan n'ont pas répondu à la demande formulée par Gorbatchev de créer une «maison commune européenne ».

11 Le territoire biélorussien a constitué le noyau de l'ancien Grand-duché de Lithuanie. Les Biélorussiens ont développé une culture spécifique combinant des éléments 
empruntés à l'Est et à l'Ouest. À partir de 1905, la paysannerie biélorussienne a en conséquence massivement adopté les idéaux égalitaristes et internationalistes prônés par les courants socialistes. Le projet soviétique a rencontré a priori en Biélorussie un terreau favorable, car il a combiné au départ promotion sociale, développement économique, internationalisme et reconnaissance de la personnalité nationale. Mais l'industrialisation à marche forcée mise en place sous Staline s'est accompagnée d'une centralisation et d'une répression qui aurait pu être fatale à l'idéal d'un "homme nouveau » soviétique si l'occupation nazie, par ses horreurs indépassables, n'avait permis à la «République des partisans » de retrouver au sortir de la Seconde Guerre mondiale une nouvelle légitimité. Malgré le retour de la centralisation et de la russification après 1945, l'épopée de la résistance massive à l'occupant et la situation stratégique occupée par la Biélorussie poussa Moscou à soutenir une politique d'investissements massifs jugée bénéfique par la masse de la population. Les anciens combattants montèrent dans la hiérarchie sociale grâce à la construction d'industries qui permirent à l'URSS de devenir une puissance militaire et spatiale. Devenue le centre des industries de pointe et « l'atelier d'assemblage de l'URSS », la Biélorussie était, à la veille de la Perestroika, fière de ses réussites. Ses habitants souhaitaient tout au plus que soit mis un terme à l'hypercentralisation du système soviétique. Dans les milieux littéraires, on était en outre choqué par les dégâts provoqués par la politique d'industrialisation à marche forcée qui avait entraîné la destruction de la vieille culture populaire, la marginalisation de la langue nationale et la destruction d'une partie de l'environnement. La catastrophe de Tchernobyl en 1986 donna à ces sentiments une vigueur nouvelle, car $70 \%$ des dépôts radio-actifs tombèrent alors sur la Biélorussie.

12 Les nationalistes biélorussiens ne réussirent cependant jamais à conquérir l'appui de la majorité de la société mais ils jouirent un temps d'une réelle légitimité. Lorsque le " putsch» de l'été 1991 se termina sur un échec lamentable, aucune force ne s'opposa plus à la rupture avec Moscou. Cette rupture partait néanmoins d'un malentendu. La fraction de l'intelligentsia qui se voulait à la fois nationaliste et pro-occidentale, souhaitait une désoviétisation complète des structures, des élites dirigeantes, de la culture et de la langue. Pour la nomenklatura et les élites « techniques» en revanche, il s'agissait d'élargir leur marge de manœuvre sans perdre l'accès aux marchés de la CEI et de se mettre à l'abri des expérimentations, dans les pays Baltes et en Pologne, ou décidées à Moscou et jugées hasardeuses. Les couches populaires quant à elles, souhaitaient des réformes prudentes permettant de maintenir la stabilité et les acquis hérités de la période soviétique.

Le parti communiste fut interdit jusqu'en 1993 mais le gouvernement du premier ministre Viatcheslav Kebitch resta en place et le pouvoir fut d'abord concentré aux mains d'une direction collégiale rassemblée dans le praesidium du Soviet suprême. Cela préserva des lieux d'arbitrage entre les différents cercles de la nomenklatura. Le refus d'instaurer en 1991 une présidence de la république s'appuya sur la légitimité soviétique originelle excluant tout pouvoir personnel. Les réformes économiques furent introduites très lentement afin d'éviter tout choc brutal. La chute de l'économie biélorussienne fut beaucoup plus lente que celle des États voisins. L'opposition nationaliste ne proposa rien d'autre que l'aventure de réformes économiques incompréhensibles, la délégitimation des engagements pris lors de la Seconde Guerre mondiale et le rejet de la langue russe qui avait permis aux couches populaires l'accès à la culture moderne universelle. 
14 L'opposition nationaliste n'a jamais exercé le pouvoir à Minsk, mais son programme « symbolique » (emblèmes, discours légitimateur, dérussification, etc.) fut adopté par le nouvel État indépendant lors de la débâcle de l'Union soviétique. La dérussification heurta une population qui, après avoir peiné à adopter le russe littéraire, éprouvait désormais une certaine condescendance à l'égard du biélorussien jugé peu utilisable comme langue de communication moderne. La Biélorussie a aussi perdu beaucoup de ses partenaires économiques, ce qui entraîna une grave dépression à partir de 1993. Tous les changements introduits après 1991 furent donc associés à la chute des conditions de vie, ce qui décrédibilisa le nationalisme et l'orientation pro-occidentale, mais aussi la nomenklatura à laquelle on reprocha de s'être maintenue aux postes de décisions sans avoir préservé la stabilité acquise au cours de la «stagnation» soviétique. Ce mécontentement poussa les élites biélorussiennes à rechercher la stabilisation des institutions en adoptant en 1994 une constitution prévoyant l'instauration d'une présidence de la république élue au suffrage universel. "Surfant » sur la nostalgie envers l'URSS, Kiebitch pensait être élu. Mais il se heurta à un nouveau venu, Alexandre Loukachenko, qui sut traduire dans un langage populaire le désarroi de l'opinion.

15 A. Loukachenko fut élu avec plus de $80 \%$ des voix au second tour. Il institua un régime autoritaire, réprimant au coup par coup les opposants de droite (nationalistes et libéraux) puis de gauche (agrariens et communistes), réhabilita certains éléments tirés de l'histoire soviétique et engagea une politique de rapprochement avec la Russie. En 1996, il organisa un référendum contesté, qui lui permit de faire plébisciter une nouvelle constitution et de nommer un nouveau parlement. Il a créé une administration présidentielle pléthorique et une "verticale», structure ramifiée chargée de faire descendre les impulsions du pouvoir. A. Loukachenko a réinstauré le contrôle gouvernemental sur les entreprises, limité la privatisation à des entreprises liées à son administration et permis l'implantation de quelques firmes occidentales voulant bénéficier de l'ordre régnant à Minsk pour pénétrer le marché russe (Coca-cola, Mc Donald's, etc.). Développant une politique de contacts tout azimut avec les régions russes, la Chine, les pays du Moyen-Orient, le régime de A. Loukachenko a permis le redémarrage de certaines branches de l'économie, en particulier l'armement, la production de tracteurs, de véhicules industriels, de téléviseurs. L'industrie biélorussienne a réussi, grâce à l'appui de l'État, à monopoliser la production pour l'exURSS d'articles autrefois concurrencés par les usines d'autres républiques ${ }^{2}$. On peut même constater une reprise de l'innovation technologique dans certaines branches. Mais la stagnation semble se poursuivre dans d'autres secteurs, ce qui accroît leur retard technologique. Pour une moitié environ de l'opinion biélorussienne et pour des secteurs importants de l'opinion russe et ukrainienne, la politique de Loukachenko a permis un redémarrage de l'économie et le maintien d'un minimum d'acquis sociaux. Pour les opposants en revanche, cette économie de commandement au jour le jour, entraînera à terme une crise encore plus profonde.

On ne pourra mesurer les résultats réels de la politique de «fraternité slave » que dans la mesure où les liens économiques qui en découlent se révéleront durables. Cette politique est possible grâce à la généralisation des accords de troc qui ont permis de contourner le manque de liquidités et les effets de la crise russe de 1998. Elle vise à reconstituer des réseaux économiques censés créer les bases d'un espace économique et commercial commun. Six oblasts de Biélorussie ont ainsi rejoint l'association 
interrégionale "Russie centrale». Les coopérations entre les villes de Minsk et de Saint-Pétersbourg sont devenues étroites ${ }^{3}$. Minsk a multiplié ses représentations officielles sur tout le territoire ex-soviétique. La perception de la Biélorussie comme territoire relativement stable est illustrée par la demande du district russe de Krasnaïa Gora d'être annexé à la Biélorussie ${ }^{4}$. Les coopérations s'étendent du niveau des entreprises à celui des régions en passant par celui de la recherche ${ }^{5}$. Mais elles se heurtent à des intérêts concurrents et aux écarts de prix. Et les prétentions de Loukachenko gênent de plus en plus Poutine.

17 À plusieurs reprises, des contrôles surprises visant à éviter les trafics ont eu lieu aux frontières entre les deux pays, malgré la création formelle d'un espace douanier commun. La grande question est de savoir si ces coopérations pourront se développer sans l'engagement permanent des administrations, ce qui nécessiterait la réapparition d'un système lourd à la soviétique refusé catégoriquement par le Kremlin. Le caractère autoritaire du régime de A. Loukachenko est incontestable, de l'avis même de son auteur qui se présente comme un patron "qui fait marcher la boutique ». Les bavures policières sont nombreuses et on note plusieurs cas de disparitions visant des opposants déclarés ou des anciens hauts fonctionnaires tombés en disgrâce. Le pluralisme persiste cependant cahin-caha, et les interdictions d'activités journalistiques n'ont jusqu'à présent jamais été définitives. Le pouvoir contrôle presque tous les médias mais n' a pas osé s'attaquer en général aux médias russes d'accès facile. Le principal handicap des forces d'opposition tient à leurs propres faiblesses et à leurs divisions. Les milieux nationalistes réussissent à mobiliser une partie de la jeunesse urbaine mais ils restent peu crédibles depuis la période 1991-1994. Les « libéraux » sont moins présents, la plupart des dirigeants d'entreprises préférant rechercher des appuis au sein de l'appareil présidentiel. L'opposition communiste jouit d'une base populaire assez large mais n'est pas habituée aux conditions de semi-clandestinité et éprouve de la difficulté à mobiliser contre un président qui réalise une partie de son propre programme (rapprochement des États post-soviétiques, maintien d'un secteur public, etc.). A. Loukachenko de son côté, n'a pas créé de grand parti unique mais s'appuie sur des partis provenant à la fois de la "gauche» postcommuniste et de la droite slavophile.

L'Ukraine : pays clef dans les stratégies des grandes puissances

La Russie utilise le territoire biélorussien pour assurer la défense de la région de Moscou et pour construire un gazoduc permettant d'accéder aux marchés occidentaux en «contournant» l'Ukraine dont les choix stratégiques restent indéterminés. La politique louvoyante menée par Kiev a longtemps gêné les maîtres du Kremlin qui savent que la possession de l'Ukraine a toujours été l'élément qui a donné à la Russie son statut de véritable puissance. L'opinion russe quant à elle, est prompte à considérer l'Ukraine comme le berceau de son identité, ce qui s'appuie sur des éléments de culture communs mais néglige les différences accumulées au cours de l'histoire entre les deux «nations-sœurs ». La situation stratégique de l'État ukrainien explique pourquoi les dirigeants de Kiev ont obtenu depuis l'indépendance des concessions de la part de Moscou et des aides de l'Occident. Les dirigeants des États-Unis ont longtemps semblé très conscients du fait que l'affaiblissement de la Russie pourrait ne pas durer et qu'il leur fallait profiter de cette conjoncture pour enraciner le séparatisme ukrainien, pour rendre impossible la résurgence à Moscou d'une puissance capable de rivaliser avec celle de Washington. Mais les conséquences des attentats du 11 septembre 2001 ont contribué à renforcer la position du Kremlin aux yeux de Washington et, en 
conséquence, à diminuer l'importance de l'Ukraine. Toutes ces questions stratégiques se heurtent aussi au fait que la société ukrainienne est fortement divisée et peu décidée à s'engager sur une voie unilatérale, dans les domaines sociaux, économiques ou géostratégiques.

$\mathrm{Au}$ début $\mathrm{du} \mathrm{xx}^{\mathrm{e}}$ siècle, les linguistes ont élaboré une identité nationale qui s'appuyait sur l'opposition des paysans ukrainiens à l'égard des seigneurs polonais ou russes. L'existence de dialectes ukrainiens leur a permis de légitimer un projet national sur la base duquel Staline donna naissance à l'Ukraine dans ses contours actuels. L'unité de la nation ukrainienne est fragile car depuis la fin du Moyen Âge jusqu'en 1944, jamais ses territoires n'ont appartenu au même ensemble politique. L'Ukraine de l'Est, du Sud puis du Centre a été progressivement détachée entre le $\mathrm{XVII}^{\mathrm{e}}$ et le $\mathrm{XVIII}^{\mathrm{e}}$ siècle de l'État polono-lithuanien et rattachée à la Russie, mais la Galicie devint autrichienne puis polonaise et ne fut rattachée à l'Ukraine qu'en 1944. Dans la partie du pays rattachée à l'empire des tsars, la politique de russification et de « retour à l'orthodoxie » a créé des différences profondes avec l'Ouest qui a pu développer une identité nationale propre en s'appuyant sur le clergé gréco-catholique maintenu par les Autrichiens. L'Ukraine orientale devint un des principaux centres industriels de la Russie tandis que l'Ouest resta rural. C'est sous Staline que se réalisa dans l'ambiguïté le rêve d'unité nationale ukrainien. L'Est et dans une moindre mesure, le Centre de l'Ukraine cultivent, malgré la famine des années trente et la politique de russification assez poussée, une nostalgie de la période soviétique qui assura l'essor de l'économie et la mobilité sociale. L'Ouest s'est barricadé au cours de la même période sur une conscience nationale, voire nationaliste, d'autant plus forte qu'il est resté plus rural, son seul grand centre urbain étant Lviv (Lvov).

20 L'Ukraine s'est engagée tardivement dans la Perestroika. Les notables de la nomenklatura reprirent à leur compte lors du démantèlement de l'URSS la légitimité nationaliste, ce qui leur a assuré une marge de manœuvre beaucoup plus grande. Malgré des divergences d'intérêts économiques ou régionaux, la nomenklatura a dans l'ensemble pu conserver ses positions car elle a défendu une ligne socio-économique "médiane", souvent qualifiée de "social-démocrate ", et visant à éviter toute cassure sociale ou régionale. Elle se présente en arbitre face aux courants néo-communistes réimplantés dans l'Est et le Sud du pays et aux nationalistes présents, malgré leur affaiblissement, à l'Ouest. Le «missionariat » patriotique des "paysans » de l'ouest ukrainien s'est en effet assez vite heurté à la résistance passive du reste du pays qui voyait son niveau de vie chuter et ignorait le sentiment nationaliste. Il existe cependant un consensus minimum qui a empêché la cassure et s'appuie sur l'identité territoriale ukrainienne issue de la période soviétique et à laquelle adhère la minorité russe ${ }^{6}$. Le passé récent (deux guerres, une guerre civile, une grande famine, plusieurs vagues de répression) et la crainte de conflits semblables à ceux qui ont traversé l'ex-URSS depuis la fin de la Perestroika ont poussé les Ukrainiens à faire montre de calme et de civisme. Les taux de participation aux élections sont en général de l'ordre de $70 \%$, ce qui est plutôt supérieur à la moyenne de l'ex-URSS.

21 Face à une opposition nationaliste et/ou pro-occidentale sans programme crédible, largement cantonnée dans l'ouest du pays et incapable de s'appuyer sur une base socioéconomique solide, deux grandes «familles » politiques ont émergé. Au «centre», les multiples clans issus de la nomenklatura soviétique se sont finalement structurés autour de Leonid Koutchma, l'ancien directeur des établissements militaires « Ioujmach » à 
Dniepropetrovsk, pépinière de cadres depuis Brejnev. Les élections présidentielles de l'automne 1999 ont démontré que, malgré la résistance des "sociaux-démocrates » liés à l'ancien chef du KGB ukrainien, Yevhen Martchouk, et à l'ancien président Leonid Kravtchouk, Koutchma avait réussi à démanteler la plupart des cercles concurrents. Les "centristes" traitent l'Ukraine comme une méga-entreprise gérée sur un mode paternaliste et négocient avec Moscou ou le FMI comme on le faisait autrefois par ses relais au Comité central. Kiev a besoin de Moscou et des régions russes pour maintenir en vie l'économie ukrainienne. Koutchma cherche à contrebalancer cette dépendance en s'appuyant sur l'UE et les États-Unis7. Sa politique "multivectorielle» de balancement lui a permis de préserver une marge de manœuvre sans pour autant enrayer la régression économique, aujourd'hui apparemment terminée avec le retour de la croissance.

La reprise se heurte à la contraction des marchés post-soviétiques et à l'absence de stratégies économiques et sociales suivies, mais l'émergence d'un secteur informel permet d'atténuer les effets de la crise. Les pouvoirs ukrainiens ont d'abord hésité à s'engager dans une politique de réformes risquant de déstabiliser le pays, d'autant plus que l'ouest du pays poussait vers le nationalisme tandis que l'Est s'y opposait. À la différence de la Biélorussie, les dirigeants de Kiev ont continué à louvoyer. Koutchma a dû composer en permanence avec les différents lobbies, les parlementaires, les nationalistes ou la gauche.

L'électorat de droite, issu du Roukh, n'a jamais réussi à dépasser $15 \%$ des suffrages. Les différentes fractions du "centre», dont le parti national-démocrate proche de Koutchma, s'appuient sur $40 \%$ environ de l'électorat. La gauche "néo-soviétique " représente la majorité relative de l'électorat avec près de $45 \%$ des voix ${ }^{8}$. Cet équilibre précaire explique à la fois les compromis quasi-permanents qui ont assuré à l'Ukraine une stabilité politique proche de la stagnation mais aussi permis la prolongation de la dépression économique. L'Ukraine n'a dans les faits toujours pas tranché entre une politique de privatisation et une relance de l'interventionnisme d'État. Les intérêts locaux, fortement représentés au parlement de ce vaste pays dépassant par sa taille celle de la France, ont souvent contribué à rendre flous les clivages entre la gauche et la droite, chaque région cherchant avant tout à obtenir des avantages de la part des pouvoirs publics et des financiers occidentaux. Les différentes régions d'Ukraine ont des intérêts économiques qui les poussent vers des stratégies de coopération différentes. L'Ouest du pays s'est rapproché de la Pologne et voit avec inquiétude les conséquences de l'intégration de la Pologne à l'espace de Schengen. Les régions de la mer Noire ont développé des coopérations avec les pays riverains. Les oblasts de l'Est restent liés à l'espace russe. Comme à l'époque soviétique mais dans un contexte de précarité accrue, les Ukrainiens «montent » vers Moscou pour faire des affaires ou chercher un travail mieux rémunéré.

La politique officielle vise à assurer un rapprochement graduel avec l'Otan et l'UE sans provoquer d'opposition frontale de la part de Moscou et sans entraîner de cassure sociale. L'Ukraine n'a jamais rompu avec la CEI mais souhaite que cette structure reste la plus inconsistante possible. Les comportements incohérents et souvent arrogants des maîtres du Kremlin depuis 1991 ont largement contribué à prolonger ce phénomène. L'Ukraine se trouve devant des choix cruciaux. Les aides occidentales n'ont pas empêché l'érosion de l'économie et les perspectives d'adhésion à l'Otan ou à l'UE sont trop lointaines pour permettre aux Ukrainiens de patienter. La guerre menée par l'Otan 
contre la Yougoslavie ou l'Irak mais aussi celle de Tchétchénie font hésiter les Ukrainiens devant les choix géostratégiques à faire. L'attentisme ne pourra cependant pas durer car l'impatience monte. L'extrême droite, dont on avait craint la résurgence, n'a pas réussi à s'incruster, ce qui montre que la propagande "internationaliste " menée pendant la période soviétique a réussi à la délégitimer. On perçoit en revanche la radicalisation de la gauche avec la montée du parti socialiste progressiste, le maintien d'un fort parti communiste et la radicalisation du Parti socialiste. Mais l'on a vu apparaître aussi un courant libéral désormais puissant autour de l'ancien Premier ministre Youchtchenko, devenu depuis président de la République et organisateur d'une réorientation stratégique tendant à pousser son pays vers le libéralisme et les pays occidentaux.

25 Les clivages historiques, régionaux et identitaires restent donc importants mais la société est de plus en plus consciente des implications qu'entraînent l'intégration de l'Ukraine dans "l'économie-monde ». Aucun gouvernement à Kiev ne peut ignorer le poids de l'Ukraine orientale pro-communiste car, malgré la désindustrialisation, cette région reste l'une des seules en état de financer le budget de l'État ${ }^{9}$. Les «aides» occidentales ont jusqu'à présent contribué à contrebalancer cette donne mais n'ont pas réussi à la modifier radicalement. L'Ukraine doit aussi assurer ses fournitures en énergie, ce qui nécessite à la fois des investissements et l'amélioration des relations avec la Russie. Le projet alternatif prôné par les États-Unis et visant à créer un réseau d'approvisionnement en énergie vers l'Europe occidentale liant à l'origine les pays du GUUAM (Géorgie, Ukraine, Ouzbekistan, Azerbaïdjan, Moldavie), et qui permettrait de contourner la Russie, nécessite des investissements considérables et la paix dans le Caucase. Il se heurte toutefois de plus en plus à l'incrédulité des pays concernés, ce qui démontre la force de l'influence russe. Il y a donc une lutte contre la montre entre les forces qui préparent le "redémarrage » de la Russie dans un monde multipolaire et celles qui tentent de créer un monde unipolaire autour des États-Unis. L'Ukraine occupe une position stratégique inconfortable mais c'est son atout principal dans sa situation de grande fragilité économique.

La lutte contre la montre comporte un autre volet. Kiev a accumulé les "aides" occidentales sans que cela n'ait donné de résultats tangibles mais il lui faudra commencer à les rembourser. L'Ukraine est aussi débitrice envers la Russie d'où elle importe presque toute son énergie. Le seul moyen pour éviter un arrêt brutal des livraisons de gaz et de pétrole dès que les voies de contournement par la Biélorussie auront été terminées, sera d'accorder à l'État ou aux entreprises russes des parts dans les entreprises ukrainiennes en cours de privatisation. Cette évolution est déjà engagée. Cela limitera à terme les marges d'autonomie de Kiev et augmentera la précarité sociale, ce qui risque de provoquer le mécontentement à la fois de la gauche et des nationalistes, mais cela contribuera aussi à pousser la Russie à négocier des accords durables avec un pays qui ne peut être que son partenaire.

Le rêve d'une indépendance totale de l'Ukraine est irréalisable, à la fois à cause de la mondialisation, des diversités internes de sa société et des liens qu'elle doit garder avec ses partenaires ex-soviétiques. Sa position stratégique donne toutefois encore un peu de temps à ses élites, ce qu'on a pu constater avec la conclusion des compromis sur le statut de la base navale de Sébastopol, sur celui de la flotte de la mer Noire et sur l'autonomie de la Crimée. Jusqu'à présent, le peuple ukrainien a fait preuve d'une grande patience et d'un esprit civique, ce que l'on mesure mal en Occident. Mais cette 
situation n'est pas éternelle et les décideurs internationaux semblent à court d'idées pour empêcher que la crise sociale et économique en Ukraine ne débouche sur une crise politique.

Moldavie, un État roumain à mi-chemin entre Bucarest et la CEI

28 Au moment du démantèlement de l'URSS, il semblait que l'État moldave était celui qui avait le moins de chance de survivre, à la fois en raison de la sécession de la Transnistrie, de la Gagaouzie et de la redécouverte des racines roumaines de la culture moldave. Les partis nationalistes roumains de Moldavie extérieure ont, dans la foulée de l'indépendance, d'abord réussi à rendre officiel le caractère "roumain » de l'État moldave et fait accepter l'idée d'une "réunification » sur le modèle de l'Allemagne un an auparavant. Mais si, sur le plan de la langue, la différence entre roumain et moldave est peu perceptible, l'histoire a provoqué des divisions qui se sont révélées durables, une fois l'euphorie de la reprise des contacts avec les "frères roumains " passée. La Bessarabie avait certes fait partie depuis le Moyen-âge de la principauté roumaine de Moldavie mais elle en avait été séparée au XIX $x^{e}$ siècle pour subir, en particulier par le biais du clergé orthodoxe russifié, l'influence d'une Russie en pleine évolution. Pendant l'entre-deux-guerres, la Bessarabie a certes été rattachée à la Roumanie, mais cette période y a laissé plutôt des mauvais souvenirs car les fonctionnaires nommés par Bucarest ont fait preuve d'arrogance et n'ont pas su organiser une politique de développement et de réformes sociales. Le rattachement de la Moldavie extérieure à l'URSS en 1940 coupa ce territoire de la Moldavie roumaine mais lui permit de devenir un des centres agricoles de "l'empire». Une nomenklatura liée au secteur agroalimentaire et aux débouchés soviétiques s'est formée depuis.

Avec la Perestroika et la chute de Ceausescu, les milieux intellectuels de la Moldavie soviétique ont commencé à rêver d'accéder, via Bucarest, au "modèle européen ». La masse de la population a découvert cependant assez vite que la Roumanie était tombée dans un état très précaire et n'était pas en mesure d'aider ses « cousins ». Les craintes d'être à nouveau traité en parent pauvre par Bucarest furent entretenues par la nomenklatura agro-alimentaire de Moldavie qui préférait l'indépendance au rattachement car cela lui permettait de jouir d'une autonomie de décision récemment acquise et de rétablir des liens économiques avec l'Ukraine et la Russie. Dès 1994, Chisinau donna au « peuple » et à la « langue » moldaves leur caractère officiel.

La question gagaouze, qui avait été soulevée avec l'appui de Moscou lors de la Perestroika, quand la Moldavie semblait tentée de regarder vers Bucarest, a été réglée pacifiquement grâce à un statut d'autonomie confirmé en 1998. La question de la région majoritairement russophone de Transnistrie est restée en revanche en suspens. Après une guerre assez courte qui a suivi le démantèlement de l'URSS, un cessez-le feu précaire continue à régner le long du Dniestr, malgré la signature d'accords prévoyant l'autonomie. La Moldavie ne peut se passer de ce territoire qui concentre les principales activités industrielles du pays et envoie $90 \%$ de ses fournitures en gaz et électricité ${ }^{10}$. Pour Moscou, la sécession larvée permet d'exercer une pression sur Chisinau tout en maintenant une base militaire sur le flanc sud-ouest de l'Ukraine.

31 Les partis pro-roumains n'ont jamais réussi à dépasser $20 \%$ des suffrages tandis que les partis « centristes » proches des différents clans de la nomenklatura se sont maintenus au pouvoir malgré la reconstitution d'un parti communiste qui est parvenu aujourd'hui au pouvoir, une première en ex-URSS depuis 1991, en obtenant la majorité absolue des suffrages. Mais ce parti n'a pas, lui non plus réussi à négocier pour le moment la paix en 
Transnistrie et se trouve toujours coincé entre une volonté de rapprochement avec le Kremlin et la nécessité de trouver des appuis en Ukraine, en Roumanie et dans l'UE.

Depuis le milieu des années 1990, les dirigeants successifs à Chisinau se sont prononcés pour l'indépendance. L'adhésion de la Moldavie à la CEI a permis d'empêcher l'unification avec la Roumanie. Seule la création d'eurorégions mises en place par le Conseil de l'Europe donne à la coopération avec la Roumanie une certaine consistance mais l'établissement d'une frontière étrange suite à l'adoption par Bucarest de réglementations européennes plus strictes a contribué à repousser, peut-être définitivement, le rapprochement entre "frères roumains». Chisinau a su aussi manifester son désir de résister à certaines pressions provenant de Moscou. Celle-ci peut jouer sur les livraisons de gaz à tarifs préférentiels et sur l'endettement de la Moldavie qui en découle. Désormais débarrassés semble-t-il du scénario d'incorporation à la Roumanie, les dirigeants de Chisinau cherchent à desserrer l'étau de la dépendance énergétique envers Moscou. Ils doivent également tenir compte des ambitions de leur voisin ukrainien, c'est pourquoi ils ont adhéré au projet du GUUAM. Face à la faiblesse de la Roumanie, au désintérêt de l'UE, l'avenir de la Moldavie dépend surtout de l'évolution des rapports russo-ukrainiens qui détermineront l'avenir de toute la région Baltique-mer Noire et sans doute de toute l'Eurasie.

La Lituanie : un État en voie de rétablissement

La république de Lituanie représente la partie de l'ancien Grand-duché de Lituanie peuplée par des populations de langue balte. En reprenant cette appellation, cet État créé en 1918 a permis à ses habitants de s'identifier à une histoire prestigieuse et d'éprouver un sentiment d'assurance faisant défaut aux autres peuples baltes et aux Biélorussiens. Proche de la Pologne par l'histoire et le catholicisme, les Lituaniens restent marqués par un fond paysan traditionnellement opposé à la culture aristocratique polonaise. Jusqu'en 1939, la Lituanie fut en conflit avec Varsovie, ce qui lui fit négliger la menace allemande et la rapprocha de Moscou. Malgré cela, le mouvement communiste est resté très faible dans ce pays de petits paysans. La chute de la Pologne en 1939 a permis à l'URSS d'annexer la Lituanie. La politique stalinienne de soviétisation brutale a heurté les Lituaniens, ce qui a facilité les compromissions de certains d'entre eux avec les nazis. Dans sa masse cependant, la société n'a ni rejoint le camp de la collaboration ni celui des résistants communistes.

L'opposition antisoviétique armée ne fut liquidée qu'au début des années cinquante. La répression entraîna alors la déportation de beaucoup de ses habitants. Malgré la faiblesse du parti communiste au départ, son premier secrétaire après 1945, Antanas Snieckus, a réussi à recruter des cadres au sein des couches montantes de la société lituanienne. Cette situation a permis la lituanisation progressive du Parti et Snieckus fit de «sa » république un fief échappant largement aux politiques homogénéisatrices et russificatrices de Moscou. La Lituanie a connu un développement industriel et agricole soutenu et a vu ses espaces d'autonomie économique et culturelle s'élargir. Après la déstalinisation en 1956 et le retour des déportés, une « petite stabilisation » a permis au Parti communiste de s'enraciner.

Au moment de la Perestroika, des cercles d'intellectuels dissidents existaient, en même temps qu'un courant majoritaire au sein du Parti communiste local se prononçait pour l'élargissement de l'autonomie de la république et l'introduction d'une certaine libéralisation de l'économie dont devaient profiter les cadres locaux. La formation du mouvement d'opposition nationaliste «Sajudis " poussa le PC local à radicaliser sa 
politique autonomiste. Seule une petite minorité voulut alors préserver l'URSS, en s'appuyant sur des fractions des minorités russes et polonaises qui craignaient le nationalisme lituanien ${ }^{11}$. Le processus d'autonomisation et de démocratisation a fait basculer la Lituanie dans l'indépendance au cours des années 1990-1991, malgré quelques tentatives maladroites de la part du Kremlin pour l'empêcher. Les élections qui ont eu lieu alors leur ont permis de légitimer l'indépendance, mais la «transition » économique qui a suivi, a entraîné de graves difficultés dont le pays n'est pas encore entièrement sorti. La "thérapie de choc ", opérée sous l'égide du président de droite, Vytautas Landsbergis, fut brutale. La décollectivisation a cassé l'ossature économique de ce pays agricole et a permis l'enrichissement d'une partie de la nomenklatura kolkhozienne. La Lituanie fut dans ce contexte, en novembre 1992, le premier pays exsocialiste à rappeler au pouvoir les "ex-communistes » du Parti démocratique du travail. Ils ont d'abord freiné le rythme des réformes capitalistes sans reconstituer pour autant les bases de «l'État-providence ». Depuis on a assisté à une série d'alternances politiques sans grands effets sur les politiques économiques générales visant à la privatisation, à l'intégration dans l'UE et à l'adhésion à l'Otan. Le "recentrage " politique opéré un temps au profit des «ex-communistes» s'est néanmoins accompagné d'une tentative de retrouver une partie des marchés ex-soviétiques.

La relative stabilisation économique s'est accompagnée de l'émergence d'un "capitalisme de réseaux» largement monopolisé par l'ancienne nomenklatura et souvent porteur de corruption. Le caractère rural, traditionaliste et catholique de la Lituanie a favorisé les changements politiques "en douceur », mais l'effondrement de l'économie a provoqué le morcellement du Sajudis, puis l'affaiblissement des «excommunistes » et la dépolitisation de l'opinion. L'interdiction du parti communiste pro-soviétique a empêché la formation d'une gauche radicale et le petit parti socialiste qui l'a remplacé n'est pas parvenu à occuper le terrain ${ }^{12}$. Malgré quelques poussées de nationalisme, le rêve d'une réussite individuelle par le biais d'une intégration à l'Occident maintient une stabilité précaire.

37 Les infrastructures du pays ont été modernisées, certains secteurs se sont développés mais des pans entiers ne se sont pas remis des "remèdes de chevaux " appliqués après 1991. Le pays a renoué avec la croissance en 1995, mais retrouve péniblement son niveau global initial. L'industrie a connu une reprise mais le secteur clef de l'agriculture a vu sa production baisser de $40 \%$ et s'enfoncer vers l'autosubsistance ${ }^{13}$. La culture et l'éducation sont dans une crise profonde. La masse de la population, y compris les classes dites « moyennes », vivent dans une précarité proche de la survie au jour le jour ${ }^{14}$. Cette situation est, aux yeux de nombreux Lituaniens, en partie compensée par le sentiment que les changements structurels réalisés et la restauration des villes et des infrastructures constituent la base d'une stabilisation permettant d'espérer dans un avenir prévisible une amélioration. La privatisation a été menée tambour battant, ce qui donne un cadre contestable mais réel pour l'organisation économique.

38 L'intégration à l'UE a été le grand dessein des élites de toutes orientations politiques. Malgré la lenteur de ce processus, le développement des échanges avec les pays occidentaux et la Pologne ont compensé en partie la perte des marchés eurasiatiques. Les perspectives de coopération avec l'enclave russe de Kaliningrad donnent un peu d'oxygène aux petits entrepreneurs qui se sont multipliés avec la décomposition et la privatisation de la plupart des grandes entreprises. 
La Lettonie : une stabilisation vulnérable

Soumise pendant plusieurs siècles à des grands propriétaires terriens allemands, la Lettonie a été assujettie tour à tour par les chevaliers teutoniques, la Pologne, la Suède et la Russie. La masse paysanne lettonne a émergé en tant que nation dans le courant $\mathrm{du} \mathrm{xIx}^{\mathrm{e}}$ siècle. Façade maritime de la Russie, les villes lettones ont connu un développement qui a permis la formation d'une bourgeoisie allemande puis d'un prolétariat letton. La naissance du nationalisme letton s'est accompagnée de l'émergence de courants socialistes et internationalistes. Le communisme a des racines en Lettonie et le nationalisme s'y est développé dans une situation de faiblesse, ce qui explique sa virulence et ses penchants fascisants manifestes pendant l'occupation nazie. La brutale politique de soviétisation et de déportations appliquée à partir de 1940 s'est accompagnée d'une forte résistance. Le parti communiste local a été marqué par une rigidité politique particulière. Il n'a finalement réussi à accueillir dans ses rangs qu'une proportion relativement faible de Lettons ${ }^{15}$. La république a joui en revanche de bonnes bases industrielles, ce qui explique que son niveau de vie était nettement supérieur à la moyenne soviétique. Cela a entraîné l'afflux d'immigrants venus de toute l'URSS, ce que les autorités locales favorisaient, voyant en cela un moyen de renforcer la formation d'un « homme nouveau » ayant « dépassé » ses identités « localistes ».

La russification fut particulièrement poussée dans les grandes villes comme Riga ou à l'Est du pays, où les Lettons devinrent minoritaires. Un courant « national » existait cependant dans le parti communiste local mais il s'est heurté à plusieurs vagues de répressions, même après la fin des purges staliniennes. La Lettonie, grâce à son niveau culturel, a exercé en revanche une influence sur l'intelligentsia russe locale qui a accueilli avec sympathie les mouvements autonomistes qui se sont manifestés lors de la Perestroika. Le "réveil national» letton s'est appuyé sur une population devenue presque minoritaire ${ }^{16}$ mais il a aussi rencontré l'appui d'une grande partie de la population russophone locale qui souhaitait se rendre autonome par rapport au centre et aux régions moins développées de l'URSS. Le Parti communiste letton s'est vite trouvé divisé entre partisans, lettons ou russes, de l'autonomie puis de l'indépendance, et fidèles de l'Union soviétique. Ces derniers recrutaient dans les milieux populaires russophones menacés par la précarisation de leurs conditions de vie, chez les militaires, nombreux dans ce pays à la vie agréable et servant de base navale, ainsi que dans une petite fraction de la population lettonne restée attachée, malgré les désillusions, aux valeurs du socialisme.

41 Les candidats du Front populaire letton ont obtenu près de $2 / 3$ des voix lors des élections de 1990, ce qui a permis le basculement vers l'indépendance dans la foulée de la désagrégation du système soviétique. Ce processus s'est appuyé sur un malentendu entre ceux qui revendiquaient l'indépendance afin de reconstruire une "Lettonie lettonne " et ceux qui penchaient pour la création d'un espace politique à base territoriale, non-ethnique, multiculturelle et économiquement plus développé. Une fois l'indépendance acquise, la citoyenneté lettonne ne fut accordée qu'à ceux qui pouvaient y prétendre au regard de la loi de 1919, ce qui a contribué à marginaliser la masse des russophones. Même si la loi a été depuis assouplie, trop de résidents restent encore apatrides ${ }^{17}$. Cette situation, combinée aux effets de l'effondrement des marchés soviétiques, a contribué à rendre passive une partie de la société. Une droite ethnonationaliste est apparue. Elle représente une menace pour l'enracinement des institutions démocratiques, d'autant plus que l'interdiction du parti communiste pro- 
soviétique et l'arrestation de plusieurs de ses dirigeants après 1991 a d'abord enlevé un contrepoids face à ces tendances. Malgré la privation du droit de vote pour une partie des russophones, un parti socialiste au programme de fait communiste a fait sa réapparition, ce qui témoigne du maintien en Lettonie des traditions prolétariennes qui semblaient avoir disparu avec la défaite des Rouges en 1919, la stalinisation après 1940 et la décommunisation après 1991. Ce phénomène explique à la fois le nombre relativement élevé en Lettonie de mariages mixtes lettons-russes et l'existence d'une réaction nationaliste particulièrement crispée.

Malgré le rétablissement économique relatif depuis quelques années, la situation de la Lettonie reste précaire, particulièrement dans les domaines sociaux, sanitaires et culturels. Une couche de la population vivant au-dessous du minimum vital est apparue. La Lettonie jouit d'un potentiel industriel traditionnel, surtout concentré dans des branches menacées par l'irruption du "capitalisme sans frontières ». La situation géographique de la Lettonie et la présence d'une forte minorité russophone lui ont permis de redevenir assez rapidement un des principaux débouchés maritimes de la Russie, en particulier pour son complexe militaro-industriel. Riga est devenue une place financière importante pour les "nouveaux Russes ». La marginalisation d'une partie de la minorité russe représente cependant un danger car cela la pousse à développer des liens avec les milieux mafieux, très présents à Riga.

La volonté des autorités lettones d'accélérer l'intégration complète de leur pays dans l'UE se heurte à la fragilité de leur économie, à la question non encore totalement réglée des droits des minorités et à la réapparition d'une droite ethno-nationaliste. Tous ces phénomènes favorisent la paralysie des institutions et retardent le plein redémarrage de l'économie. La Lettonie, plus encore que ses voisins, a besoin de prendre en compte les données géographiques qui exigent que l'on trouve un moyen d'intégrer la Russie au processus de construction d'une nouvelle architecture de l'ensemble du continent européen.

L'Estonie : un frêle petit dragon

Depuis son indépendance, l'Estonie a souvent été présentée comme un exemple dans les médias occidentaux à la recherche de bons élèves de la "transition" vers le capitalisme. Des trois pays baltes, c'est le plus petit et celui dont l'intégration à l'UE pose le moins de problèmes. C'est aussi celui qui a connu le rétablissement le plus perceptible.

45 Cousins des Finnois, les Estoniens ont subi l'influence historique de la Scandinavie et de l'Allemagne. Comme les Lettons, les Estoniens ont formé jusqu'à la Première Guerre mondiale un peuple paysan soumis aux «barons baltes» d'origine allemande. Le mouvement estonien à l'époque des tsars a d'abord été dirigé contre le germanisme avant de s'opposer à la politique russificatrice entreprise à la veille $d u x^{e}$ siècle. De 1917 à 1922, le pays a connu un conflit entre les germano-baltes, les indépendantistes estoniens et les communistes locaux qui ont fait montre d'un radicalisme révolutionnaire très poussé. L'annexion à l'URSS se fit en 1940 et a rencontré certains appuis dans la société qui est restée cependant dans son ensemble attachée au souvenir de l'État indépendant dénigré de façon simpliste par la propagande soviétique. Comme dans les autres pays baltes, une couche de collaborateurs est apparue au cours de l'occupation nazie. Après 1944, un mouvement clandestin antisoviétique a persisté quelques années. Il fut combattu par une dure répression et par la déportation de nombreux Estoniens. Au sein du Parti communiste 
estonien, une tendance autonomiste a persisté malgré les vagues d'épuration. Des courants dissidents sont apparus dans la république au cours des années 1980. La formation du Front populaire dès le début de la Perestroika a favorisé le renforcement des communistes autonomistes et la proclamation de la souveraineté de la république dès novembre 1988. Les élections de 1989 ont vu la victoire des candidats du Front populaire et le début de la marche vers l'indépendance.

Comme dans les autres pays baltes, le niveau de vie était supérieur à la moyenne soviétique et l'atmosphère plus ouverte, ce qui a favorisé l'immigration de populations russophones, particulièrement dans les villes de l'est du pays où elle sont devenus majoritaires. Une partie importante de cette minorité s'est prononcée pour l'indépendance et, comme en Lettonie, elle fut privée du droit à la citoyenneté estonienne une fois l'indépendance acquise ${ }^{18}$.

Après une dépression économique qui avait entrainé une chute de $40 \%$ du PIB, la reprise s'est manifestée dès 1994 avec des taux de croissance dépassant $5 \%$ par an ${ }^{19}$. Aux yeux des dirigeants de l'UE, l'Estonie est un des pays les plus sérieux parmi les nouveaux adhérents. Le pays s'est ouvert aux pays occidentaux, en particulier à la Scandinavie, mais il reste un débouché important pour l'économie russe. Comme en Lettonie, cette situation, combinée avec le sentiment de marginalisation ressenti par la minorité russophone concentrée dans les régions frontalières, favorise les trafics et l'implantation des réseaux mafieux. C'est dans les régions de l'Est que, lors des élections locales auxquelles peuvent participer les résidents non-citoyens, les partis russes ou le Parti démocratique du travail issu de l'aile indépendantiste du Parti communiste obtiennent la majorité des voix.

La décomposition et la recomposition du tissu social en Estonie ont permis l'émergence de nouvelles couches sociales et de nouvelles formations politiques. Elles ont également favorisé la dépolitisation, la corruption et l'émergence de réseaux mafieux. Les catégories sociales marginalisées d'origine russe sont tentées par le nationalisme russe ou le néo-communisme, tandis que beaucoup d'Estoniens "de souche » penchent vers l'ethno-nationalisme, d'autant plus que l'interdiction du parti communiste a empêché la formation d'une gauche radicale. Dans l'ensemble cependant, c'est «l'alternance sans alternative " qui s'est instaurée entre des partis de centre-droit et de centregauche, dont les orientations politiques diffèrent peu. La scène économique, sociale et politique reste dominée par des clans dont les rivalités intéressent peu la population. Comme dans les autres pays de la région, une course contre la montre semble engagée entre les forces qui organisent la recomposition de la vie sociale et économique et tablent sur la stabilisation dans le cadre de l'UE et celles qui s'appuient sur le désespoir des catégories marginalisées.

Un désir frustré de stabilité

Le calme qui a régné globalement depuis 1991 dans l'espace Baltique-mer Noire n'est pas dû au fait que les populations locales sont satisfaites par la façon dont les affaires ont été gérées ou par les politiques de réformes, là où elles ont été menées. Ce sont plutôt les conflits sanglants dans l'ex-URSS et la précarisation de pans entiers de ces sociétés consacrant désormais leur temps à des activités de survie qui expliquent la passivité relative actuelle. Après les drames vécus au $\mathrm{xx}^{\mathrm{e}}$ siècle, ces sociétés désirent avant tout la stabilité et la sécurité. C'est pourquoi, après la décomposition de l'Union soviétique, les regards se sont tournés vers l'UE, qui apparaissait comme un havre de paix et de prospérité. Seuls les pays baltes ont reçu d'Occident les signaux leur 
permettant d'espérer accéder rapidement à cette stabilité, après avoir introduit des réformes socialement douloureuses. L'Otan a en revanche été perçu au départ, sauf dans les pays baltes, comme une structure archaïque héritée de la guerre froide. Mais les guerres dans l'ex-URSS ont rendu dans les pays baltes et chez certains Ukrainiens une seconde légitimité à l'Otan. Celle-ci a cependant été largement compromise dans les pays de tradition slave-orthodoxe avec l'attaque contre la Yougoslavie du printemps 1999 qui a fait resurgir les images laissées par la propagande soviétique. Même les pays baltes, qui auraient pu envisager de voir dans l'adhésion à l'Otan une garantie donnée aux investisseurs occidentaux, doivent désormais tenir compte du fait que la Russie s'oppose à son élargissement vers son "étranger proche », ce qui pourrait menacer le développement de contacts économiques indispensables au développement de l'ensemble de la région.

Après l'histoire et une lecture sélective de son passé, la géographie reprend partout ses droits et, avec elle, le souvenir contrasté et contradictoire de l'héritage soviétique qui réapparait sous un jour nouveau. Les sociétés de l'espace Baltique-mer Noire ne peuvent plus accepter sans hésitations le «modèle occidental » qui reste cependant pour le moment sans alternative, même s'il a perdu beaucoup de son clinquant. Les sociétés de l'espace Baltique-mer Noire commencent à ressentir le besoin de faire un inventaire approfondi et objectif du passé soviétique et pré-soviétique. La formation de tissus sociaux modernes, l'enracinement des valeurs de solidarité et d'internationalisme constituent un garde-fou contre les tendances individualistes et archaïques ou ethno-religieuses qui ont réémergé dans la foulée de la décrédibilisation et du démantèlement de l'Union soviétique. Pour la première fois depuis des siècles, les populations de l'espace Baltique-mer Noire renouent avec la possibilité de pouvoir choisir leurs partenaires sans avoir trop à compter avec les pressions des grandes puissances. Ce répit permettra-t-il l'élaboration d'un modèle de développement équilibré, stable et démocratique? Quelle sera la place réellement occupées par les questions identitaires dans une situation marquée par la prééminence des questions économiques et sociales?

51 L'évolution au cours des dernières années dans le monde et l'espace Baltique-mer Noire aura permis de clarifier la situation dans une certaine mesure. Les trois pays baltes ont finalement adhéré à l'Otan et à l'UE. Ce processus a bénéficié d'un consensus non dénué de doutes grandissants mais il s'est réalisé. Depuis, ces pays semblent avoir redécouvert qu'ils constituent aussi la façade maritime de la Russie et semblent de plus en plus intéressés à jouer un rôle d'intermédiaire, même si les causes de tensions avec Moscou n'ont pas toutes disparu : intégration des minorités russes, problèmes du transit pour la région de Kaliningrad, assouplissement inéluctable des règles du traité de Schengen, difficulté à trouver des compromis avec les intérêts stratégiques de la Russie tout en subissant les pressions pour devenir une base permanente de l'aviation des États-Unis en direction de l'Asie centrale, place de la souveraineté nationale dans une UE en voie de fédéralisation, etc.

52 Les trois autres pays de cet espace, Biélorussie, Ukraine, Moldavie extérieure, quant à eux, se sont vus privés de perspectives rapides en direction de l'Ouest, ce qui a renforcé le poids de ceux qui, issus de la nomenklatura, commencent à estimer que l'appui de Moscou redevient la seule solution pour garantir la pérennité des institutions et de ceux qui en sont les premiers à en tirer profit. Mais l'émergence difficile d'un « capital national » local, souvent en concurrence avec le capital transnational basé en Russie ou 
d'un capital national lié aux relations commerciales avec l'espace post-soviétique, provoque aussi des contradictions qui expliquent pourquoi les milieux libéraux d'opposition restent divisés sur les questions géo-stratégiques.

C'est dans ce contexte général qu'il faut analyser la prolongation des questions identitaires qui recouvrent, au nom d'un hier souvent mythifié, des réalités concrètes auxquelles on veut répondre parfois ou que l'on veut parfois aussi fuir. Mais la masse des sociétés concernées ne peut pas fuir la réalité comme c'est le cas de certains intellectuels, et elle est donc amenée petit à petit à redécouvrir non seulement l'identité et l'histoire, mais aussi la géographie et la géo-économie. La stabilisation de l'espace Baltique-mer Noire comme zone d'échange entre l'Europe occidentale et l'Extrême-Orient semble engagée après les soubresauts "occidentalistes » et « eurasiens » du xxe siècle.

\section{NOTES}

1. I. Chevtsov, «L'espace mer Baltique-mer Noire: des voies divergentes de développement économique ", dans Nouveaux mondes, $\mathrm{n}^{\circ} 9$ : Les confins de l'OTAN l'espace mer Baltique-mer Noire, CRES, Genève 1999, p. 131.

2. Idem p. 142. En 1997, la Biélorussie produisait $95 \%$ des microprocesseurs de l'exURSS, $70 \%$ des tracteurs et des postes de télévision, $60 \%$ des camions lourds, alors que les usines de tracteurs de Kharkiv en Ukraine, de Lipietsk et de Tchéliabinsk en Russie ou celle de Camions du Tatarstan sont quasiment arrêtées.

3. J.-C. Lallemand, « De L'union russo-biélorussienne à l'union "à trois" avec la Yougoslavie?", Nouveaux Mondes, op. cit., p. 180.

4. Idem, p. 189.

5. Les universités de Smolensk et de Vitebsk ont par exemple fusionné au sein d'une « Université slave », J-C. Lallemand, op. cit., p. 190.

6. Les Russes représentent $22 \%$ de la population qui est par ailleurs russophone à $40 \%$. 7. L'UE est le principal bailleur de fonds de l'Ukraine et les États-Unis, dont les volumes « d'aides » internationales sont nettement moindres, accordent à Kiev la troisième place, après Israël et l'Egypte, dans ses choix.

8. La gauche ukrainienne s'appuie sur trois principaux partis, dont le parti socialiste, qui s'est constitué après l'interdiction du parti communiste en 1991, le parti communiste qui s'est reconstitué dès que la loi l'a permis et le parti socialiste progressiste, scission récente et ultra-gauchiste du parti socialiste.

9. I. Chevtsov, op. cit., p. 134.

10. I. Dinu, «La Roumanie et ses voisins de l'ex-URSS », Nouveaux Mondes, op. cit., p. 227.

11. Les districts polonais entourant Vilnius ont d'abord tenté de rester au sein de l'URSS puis, après 1991, de créer des régions autonomes, ce qui a provoqué l'arrestation de plusieurs dirigeants communistes ou polonais.

12. Sur le morcellement des partis politiques dans les pays baltes, voir Bruno Drweski, "Les paysages politiques du postcommunisme ", dans Les territoires centre-européens Dilemmes et défis - l'Europe médiane en question, La Découverte 1998, p. 76-77. 
13. Yves Plasseraud, Les États baltes, Montchrestien, 1996, p. 124.

14. Le niveau de vie aurait chuté de $80 \%$ entre 1991 et 1995, mais l'on estime que le secteur « gris » représente une soupape de sûreté qui compte pour un tiers de l'activité économique. Voir Y. Plasseraud, op. cit.

15. En 1990, seuls $40 \%$ de ses membres étaient d'origine lettone. Y. Plasseraud, op. cit., p. 71.

16. Les Lettons représentaient $52 \%$ de la population en 1989.

17. Les russophones représentent aujourd'hui $45 \%$ environ de la population.

18. Il y avait encore $38 \%$ de personnes n'ayant pas reçu le statut de citoyens en 1993, chiffre qui a tendance à diminuer avec l'assouplissement des législations. Y. Plasseraud, op. cit., p. 38.

19. Y. Plasseraud, op. cit., p. 42.

\section{RÉSUMÉS}

L'isthme situé entre la mer Baltique et la mer Noire constitue un espace géographique de transition reliant la masse continentale de l'Eurasie à la péninsule européenne. Croisement de langues et de religions différentes, il a formé pourtant une entité politique commune entre le XIV et le $\mathrm{xVIII}^{\mathrm{e}}$ siècle. Tous ces éléments historiques ont constitué les fondements de différentes identités nationales et d'habitudes sociales connaissant à l'heure actuelle des mutations profondes sous les effets contradictoires de l'indépendance nationale et de la mondialisation.

Bruno Drweski. Baltic and Black sea space

The isthmus situated between the Baltic sea and the Black sea forms an intermediary geographical area linking the Eurasian heartland with the European peninsula. Being situated at the crossroad of different languages and religions, this area has yet formed a common political entity from the $\mathrm{xIV}^{\text {th }}$ century up to the $\mathrm{xVIII}^{\text {th }}$ century. All these historical aspects gave the foundations for the different national identities and social habits that are now facing profound changes linked with the contradictory effects of the national independance and the globalization.

\section{AUTEUR}

\section{BRUNO DRWESKI}

Maître de conférences à l'Inalco, HDR, bruno.drweski@wanadoo.fr 